\title{
Tool wear detection with fuzzy classification and wavelet fuzzy neural network
}

\author{
Yingxue Yao*, Xiaoli Li, Zhejun Yuan \\ Department of Mechanical Engineering, Harbin Institute of Technology, Harbin 150001, People's Republic of China
}

Received 31 March 1998; received in revised form 22 January 1999

\begin{abstract}
In the paper, a new method of tool wear detection with cutting conditions and detected signals is presented, which includes the model of wavelet fuzzy neural network with acoustic emission (AE) and the model of fuzzy classification with motor current. The results of tool wear estimated by cutting conditions and detected signals (spindle motor current, feed motor current and AE) are fused by fuzzy inference. Experimental results show that the method of tool wear detection is reliable and practical. (C) 1999 Elsevier Science Ltd. All rights reserved.
\end{abstract}

Keywords: Tool wear; Wavelet fuzzy neural network; Fuzzy inference

\section{Introduction}

In flexible manufacturing systems (FMS), tool wear detection plays a critical role in dictating the dimensional accuracy of the workpiece and guaranteeing an automatic cutting process. It is therefore essential to develop simple, reliable and cost-effective on-line tool wear detection methodologies. Various methods for tool wear monitoring have been proposed in the past. Motor current and $\mathrm{AE}$ are considered as the most effective means of sensing tool wear. The measuring apparatus of motor current method does not disturb the machining process. Moreover it can be applied in the manufacturing environment at almost no extra cost [1]. The major advantage of using $\mathrm{AE}$ to detect tool condition is that frequency of the AE signal is much higher than that of

* Corresponding author. E-mail: yxyao@hope.hit.edu.cn 
the machine vibrations and environmental noises, and that there is no interference with the cutting operation [2-5].

In the paper, measured spindle motor current, feed motor current and AE signals are used to estimate the tool wear states. It is known that current signals depend on the cutting variable, the cutting speed $v$, the feed rate $f$, the depth of cut $d$, as well as on the tool wear $w$. This paper introduces a new method to estimate tool wear states from current measurements by the neural network model with regression technology and fuzzy classification over a wide range of cutting conditions. Wavelet transform has a good resolution in frequency and time domain synchronously. It can extract more information in the time domain at different frequency bands [6]. The wavelet packet transform can decompose a sensor signal into different components in different time windows and frequency bands. The components, hence, can be considered as the features of the original signal [7]. In the paper, wavelet packet transform is used to capture important features of the AE signal that are sensitive to the changes of tool states. A fuzzy neural network model is proposed to describe the relationship between the extracted features and tool wear states [8]. Finally, a fuzzy fusion method is presented to fuse the detection results with spindle current, feed current and AE signals. Experimental results show that the above method can be effectively employed in practice.

\section{Tool wear detection with fuzzy classification}

\subsection{The model}

Based on studies, it is suggested that the effects of tool wear, spindle speed, feed rate and the depth of cut should be taken into account when modeling current signals. In the paper, the tool wear states were divided into A, B, C, D, E, F classifications as shown in Table 1. The models of spindle and feed current as functions of spindle speed $v(\mathrm{~m} / \mathrm{min})$, feed speed $f(\mathrm{~mm} / \mathrm{rev})$ and the depth of cut $d(\mathrm{~mm})$ under different tool wear classifications are established, respectively.

The effects of the cutting variables $v, f$, and $d$ on the spindle and feed current signals under different tool wear classifications can be expressed as follows:

$$
I_{S}=K_{S} \nu^{a_{1}} f^{a_{2}} d^{a_{3}}, I_{F}=K_{F} \nu^{b_{1}} f^{b_{2}} d^{b_{3}}
$$

where $I_{S}$ and $I_{F}$ are spindle and feed current amplitude, respectively; $K_{S}$ and $K_{F}$ are constants with the tool geometry and workpiece material. If Eq. (1) is taken by logarithm, the results under A, B, C, D, E, F classifications are put in order as follows:

Table 1

Classification of tool flank wear

\begin{tabular}{lllllll}
\hline Classification & $\mathrm{A}$ & $\mathrm{B}$ & $\mathrm{C}$ & $\mathrm{D}$ & $\mathrm{E}$ & $\mathrm{F}$ \\
\hline $\begin{array}{l}\text { Tool wear } \\
\text { value }(\mathrm{mm})\end{array}$ & $0.0-0.2$ & $0.1-0.3$ & $0.2-0.4$ & $0.3-0.5$ & $0.4-0.6$ & $0.5-1.0$ \\
\hline
\end{tabular}




$$
\begin{gathered}
{\left[\begin{array}{c}
S_{1} \\
S_{2} \\
S_{3} \\
S_{4} \\
S_{5} \\
S_{6}
\end{array}\right]=\left[\begin{array}{llll}
a_{10} & a_{11} & a_{12} & a_{13} \\
a_{20} & a_{21} & a_{22} & a_{23} \\
a_{30} & a_{31} & a_{32} & a_{33} \\
a_{40} & a_{41} & a_{42} & a_{43} \\
a_{50} & a_{51} & a_{52} & a_{53} \\
a_{60} & a_{61} & a_{62} & a_{63}
\end{array}\right] \cdot\left[\begin{array}{c}
1 \\
\lg \nu \\
\lg f \\
\lg d
\end{array}\right]} \\
\text { and }\left[\begin{array}{l}
F_{1} \\
F_{2} \\
F_{3} \\
F_{4} \\
F_{5} \\
F_{6}
\end{array}\right]=\left[\begin{array}{llll}
b_{10} & b_{11} & b_{12} & b_{13} \\
b_{20} & b_{21} & b_{22} & b_{23} \\
b_{30} & b_{31} & b_{32} & b_{33} \\
b_{40} & b_{41} & b_{42} & b_{43} \\
b_{50} & b_{51} & b_{52} & b_{53} \\
b_{60} & b_{61} & b_{62} & b_{63}
\end{array}\right] \cdot\left[\begin{array}{c}
1 \\
\lg \nu \\
\lg f \\
\lg d
\end{array}\right]
\end{gathered}
$$

where $S_{i}, F_{i}(i=1,2, \ldots 6)$ are the logarithm values of the spindle current $I_{S}, I_{F}$, respectively, $\lg$ represents the usual logarithm. The above matrix relationship between the current and cutting parameters can be expressed by a neural network topological diagram (see Fig. 1). It is obvious that the input variables of the neural network are $1, \lg v, \lg f$, and $\lg d$, the output variables of that are $S_{i}, F_{i}(i=1,2, \ldots 6)$, the weights of the neural network are calculated by regression technology.

\subsection{Fuzzy classification}

Spindle current signal and feed current signal models at the different wear states are established, respectively. The models can then be used to estimate the tool wear states by a known spindle current signal, feed current signal and cutting parameters.

Measured current $S_{0}, F_{0}$ are defined as real feature values. Estimated current values $S_{i}, F_{i}$ $(i=1,2, \ldots 6)$ are defined as the cluster centers of different tool wear classifications. Real feature values $S_{0}, F_{0}$ are compared with the estimated features $S_{i}, F_{i}$ by the fuzzy classification method, the membership degrees of different tool wear classification are calculated by Fig. 2. The same method is fitted to feed current.

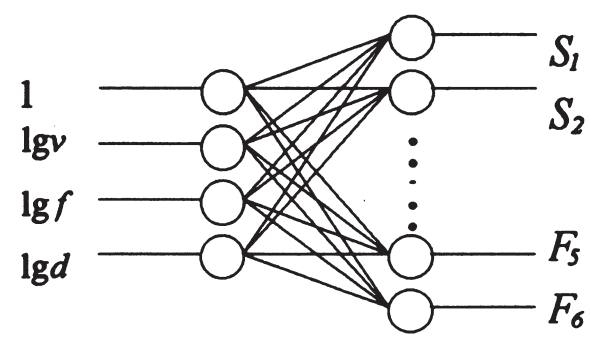

Fig. 1. Model of current with neural network. 


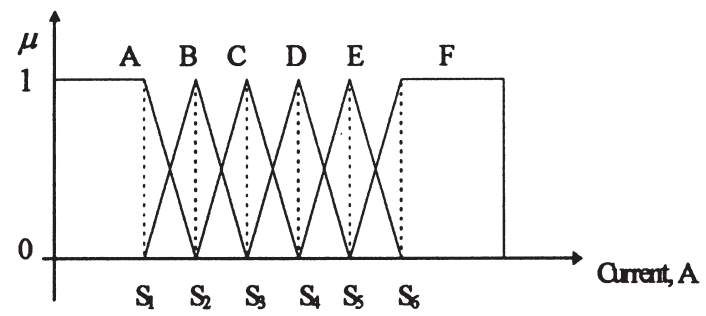

Fig. 2. Curve of membership degree.

\section{Feature of AE signal with wavelet packet transform}

\subsection{Wavelet packet transform}

Given a time varying signal $f(t)$, wavelet transforms (WT) consist of computing coefficients that are inner products of the signal and a family of wavelets. In a continuous wavelet transform (CWT), the wavelet corresponding to scale $a$ and time location $b$ is

$$
\psi_{a, b}=\frac{1}{\sqrt{|a|}} \psi\left(\frac{t-b}{a}\right) a, b \in R, a \neq 0
$$

Where $a$ and $b$ are the dilation and translation parameters, respectively. The continuous wavelet transform was defined as follows:

$$
w_{f}(a, b)=\int x(t) \psi_{a, b}^{*}(t) \mathrm{d} t
$$

Where '*' denotes the complex conjugation. With respect to $w_{f}(a, b)$ a signal $f(t)$ can be decomposed into

$$
f(t)=\frac{1}{c_{\psi}} \int_{-\infty}^{+\infty} \int_{0}^{+\infty} w_{f}(a, b) \frac{1}{\sqrt{|a|}} \psi\left(\frac{t-b}{a}\right) \mathrm{d} a \mathrm{~d} b
$$

where $c_{\psi}$ is a constant depending on the base function. Eq. (5) implies that WT can be considered to $f(t)$ signal decomposition. The WT is a time-frequency function which describes the information of $f(t)$ in various time windows and frequency bands.

Wavelet packets are particular linear combinations of wavelets. They form the bases that retain many of the orthogonality, smoothness and location properties of their parent wavelet [9]. The coefficients in the linear combinations are calculated by a factored or recursive algorithm with the result that expansions in the wavelet packet bases have low computational complexity.

The discrete wavelet packet transform can be written as follows:

$$
c_{j}[f(t)]=H\left\{c_{j-1}[f(t)]\right\}+G\left\{d_{j-1}[f(t)]\right\} d_{j}[f(t)]=G\left\{c_{j-1}[f(t)]\right\}+H\left\{d_{j-1}[f(t)]\right\}
$$

where $H$ is the analysis discrete wavelet, $G$ is the scaling sequence, $c_{j}$ is the approximation of $f(t), d_{j}$ is the detail signal of $f(t)$. Let $Q_{j}^{i}(t)$ be the $i$ th packet on $j$ th resolution, then, the wavelet packet transform can also be computed by the recursive algorithm below: 


$$
Q_{0}^{1}(t)=f(t), Q_{j}^{2 i-1}(t)=H Q_{j-1}^{i}(t) Q_{j}^{2 i}(t)=G Q_{j-1}^{i}(t)
$$

where $t=1,2, \ldots 2^{J-i}, i=1,2,2^{j}, j=1,2, \ldots, J, J=\log _{2} N, N$ is data length.

\subsection{AE signal pretreatment}

Research has shown that AE, which refers to stress waves generated by the sudden release of energy in deforming materials, has been successfully used in laboratory tests to detect tool wear and fracture in single point turning operations [10]. We have found that friction and plastic deformation have comparable importance with regard to the generation of the continuous AE. In the case of detecting AE signals from a tool bar in a similar way as shown in Fig. 7, the amplitude of the AE signals from the workpiece is reduced as the wave transfers from workpiece to tool at the interface. The relationship between the RMS of continuous AE and the cutting parameters as well as tool wear can be established by experimental methods. Results have shown that its RMS is proportional to $v_{\mathrm{c}} a_{\mathrm{p}}$, tool flank wear and $\mathrm{VB}$, respectively, but it is independent of feed rate. According to experimental results, the RMS of AE can be calculated from the machining and tool wear parameters:

$$
\mathrm{RMS}=K v_{c} a_{p} \mathrm{VB}
$$

where $K$ is the area density of contact points, $v_{c}$ the cutting speed, $a_{p}$ the depth of cut, VB the wear land. $K$ depends on the structure of the surface, which remains almost constant with increasing wear.

During the experiments, the friction between workpiece and tool generates a continuous AE signal which gives information on tool wear. However, the experimental results show that sometimes burst signals with high peak amplitudes interfere with the continuous AE signal. In fact, these burst signals relate to the chip breakage, give information on the chip behavior, but not on tool wear. Therefore, it is essential to filter out these bursts from the continuous AE signal, for reliable tool wear monitoring, before further analysis is performed. The floating threshold value is defined, which is higher than the mean signal level. The constituents of chip impact and breakage exceeding this threshold are not considered as the determination of the mean signal level and are filtered out from the continuous AE signal. The signal constituents below the threshold represent the continuous AE, which will be analyzed by the following signal processing method.

\subsection{Signal analysis and features extraction}

In monitoring of tool wear, AE signals monitored contain complicated information on the cutting processing. To ensure the reliability of a tool monitoring system, it is important to extract the features of the signals.

Fig. 3 shows typical changes of AE signals in the boring process. The AE signals in a time domain are presented. At the beginning of the cutting process the signal affected by tool wear is smaller because the tool is fresh, the magnitude of the AE is small and the cutting process is stable. As tool wear increases, the magnitudes of the AE increase.

Fig. 4 shows the decomposing results of AE signal for the experiment shown in Fig. 3 through wavelet packet decomposition. The frequency windows and their bands are indicated in Table 2. 


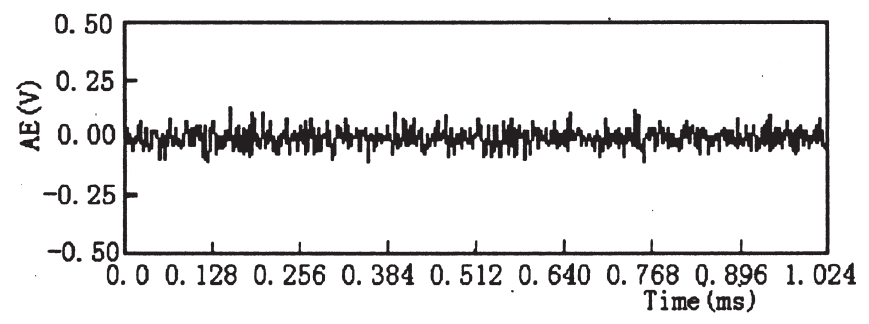

(a) $V B=0.06 \mathrm{~mm}$

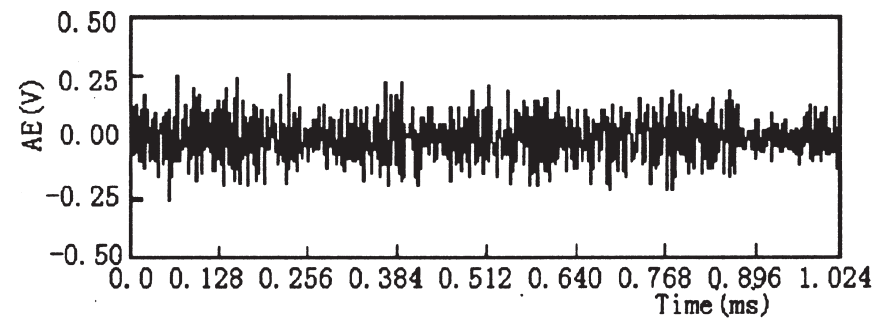

(b) $V B=0.26 \mathrm{~mm}$

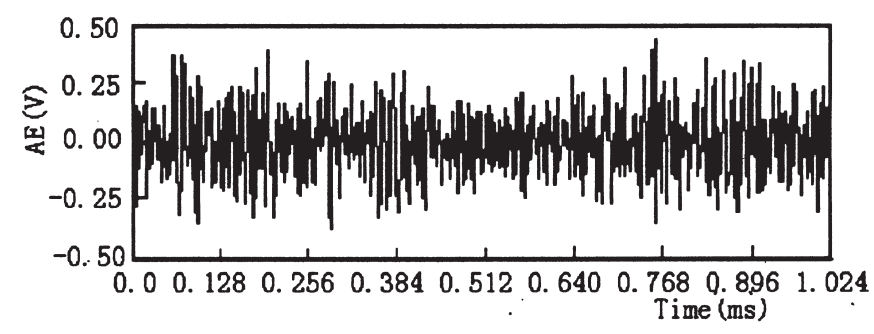

(c) $V B=0.62 \mathrm{~mm}$

Fig. 3. AE signals in a typical tool wear cutting process. Cutting speed: $30 \mathrm{~m} / \mathrm{min}$; feed rate: $0.2 \mathrm{~mm} / \mathrm{rev}$; depth of cut: $0.5 \mathrm{~mm}$. Workpiece material: $40 \mathrm{Cr}$ steel; tool material: high-speed-steel, without coolant.

Obviously, these decomposed results of the AE signal not only keep the same features as discussed above, but also provide more information, such as the time domain constituent parts of the $\mathrm{AE}$ signal at the frequency band. The mean values of the constituent parts of the AE of each frequency band can represent the energy level of the AE in the frequency band.

For tool wear monitoring, the RMS in each frequency band was used to describe the features of different tool condition. The selected features were summarized as follows:

$n_{1}=$ RMS of wavelet coefficient in the window 1

$n_{2}=$ RMS of wavelet coefficient in the window 2

$\vdots: \vdots$

$n_{16}=$ RMS of wavelet coefficient in the window 16

But not all of the above features are sensitive to tool wear. According to a large amount of data analysis, only $n_{3}, n_{4}, n_{5}, n_{6,} n_{7}, n_{8}$ and $n_{13}$ are sensitive to tool wear, Figs. 5 and 6 show two typical examples, where the above features are replaced by $q_{1}, q_{2}, q_{3}, q_{4}, q_{5}, q_{6}, q_{7}$, respectively, and will be used to classify tool wear states. 


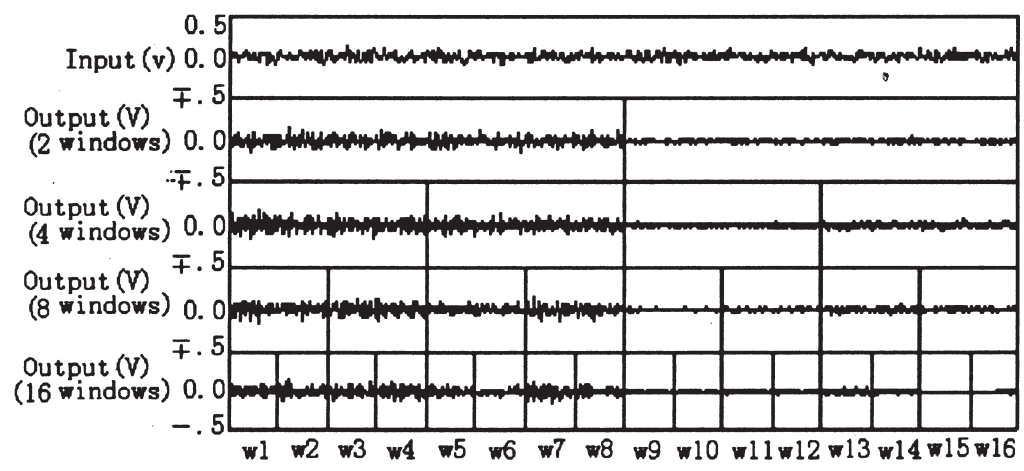

(a) $\mathrm{VB}=0.06 \mathrm{~mm}$

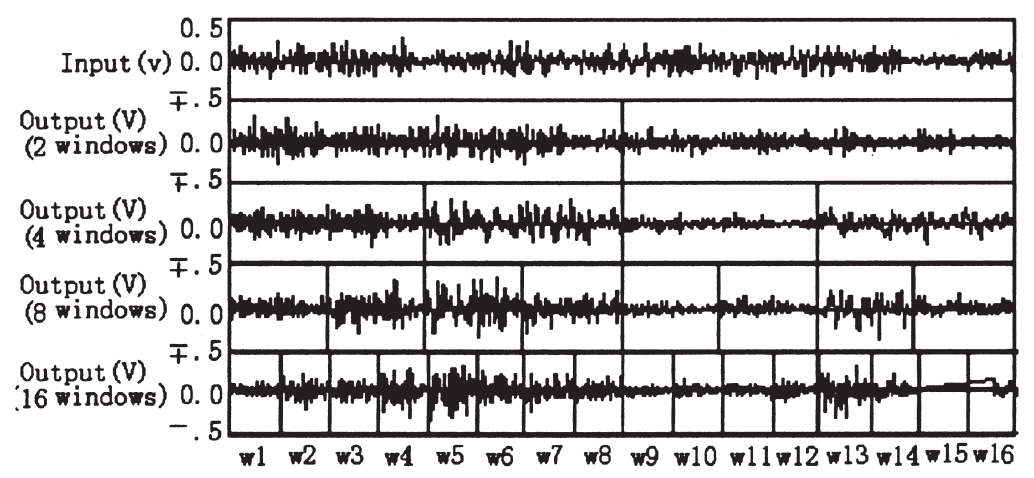

(b) $V B=0.26 \mathrm{~mm}$

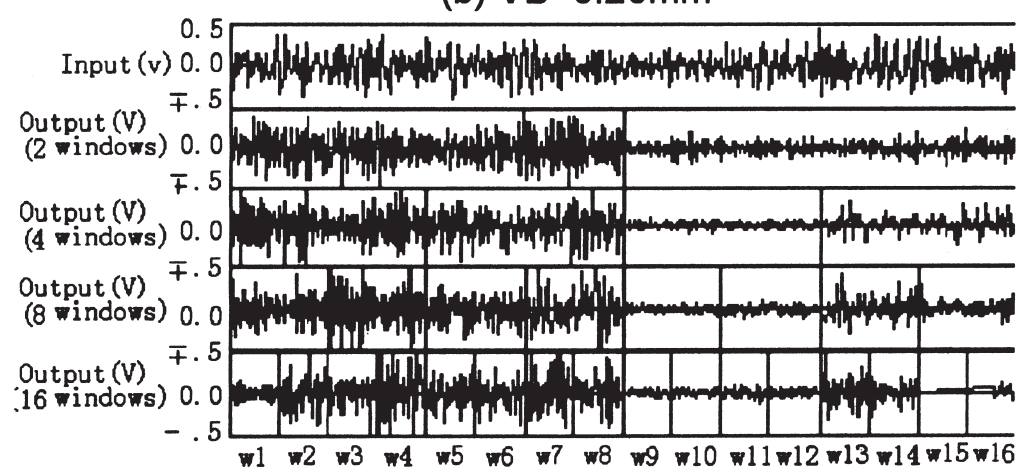

(c) $V B=0.62 \mathrm{~mm}$

Fig. 4. Decomposing results of AE by wavelet packet transformation.

According to Eq. (8), it shows that RMS of the continuous AE is proportional to $v_{\mathrm{c}}, a_{\mathrm{p}}$ and tool flank wear VB, but it is independent of feed rate. To eliminate the effects of cutting conditions on features, $q_{\mathrm{i}}(i=1,2, \ldots, 7)$ is divided by $v_{\mathrm{c}} a_{\mathrm{p}}$ to obtain new $q_{\mathrm{i}}$ values, the new $q_{\mathrm{i}}$ values are the final monitoring features. 
Table 2

Windows and their frequency bands

\begin{tabular}{ll}
\hline Window no. & Frequency band \\
\hline 2 & W1: $0-0.5 \mathrm{MHz}$ \\
& W2: $0.5-1 \mathrm{MHz}$ \\
4 & W1: $0-0.25 \mathrm{MHz}$ \\
& W2: $0.25-0.5 \mathrm{MHz}$ \\
& $\downarrow$ \\
& W4: $0.75-1.0 \mathrm{MHz}$ \\
8 & W1: $0-0.125 \mathrm{MHz}$ \\
& W2: $0.125-0.25 \mathrm{MHz}$ \\
& $\downarrow$ \\
& W8: $0.875-1.0 \mathrm{MHz}$ \\
16 & W1: $0-62.5 \mathrm{kHz}$ \\
& W2: $62.5-125 \mathrm{kHz}$ \\
& $\downarrow$ \\
& W16: $0.9375-1.0 \mathrm{MHz}$ \\
\hline
\end{tabular}

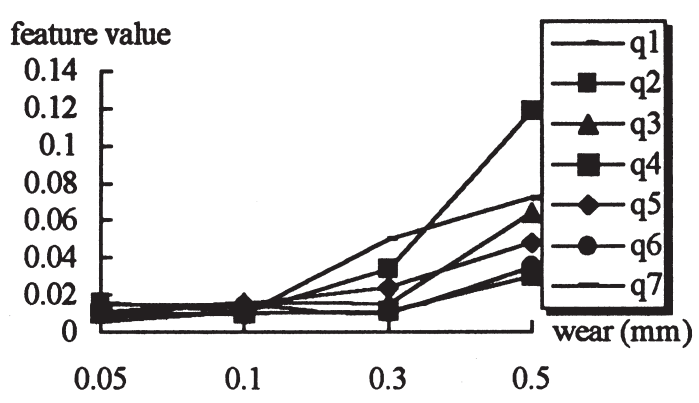

Fig. 5. The relationship between features extracted and tool wear. Cutting speed: $30 \mathrm{~m} / \mathrm{min}$; feed rate: $0.2 \mathrm{~mm} / \mathrm{rev}$; depth of cut: $0.5 \mathrm{~mm}$. Workpiece material: $40 \mathrm{Cr}$ steel; tool material: high-speed-steel, without coolant.

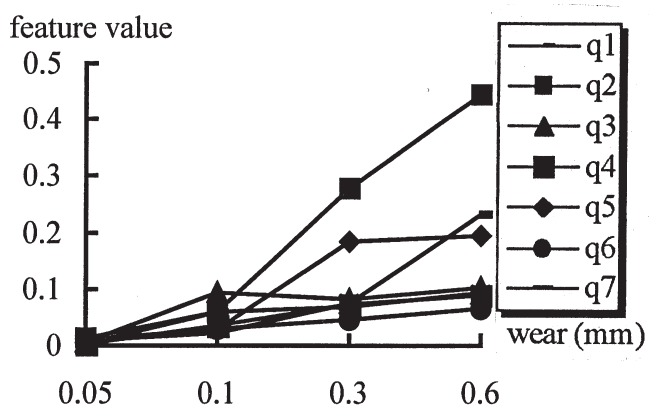

Fig. 6. The relationship between features extracted and tool wear. Cutting speed: $40 \mathrm{~m} / \mathrm{min}$; feed rate: $0.3 \mathrm{~mm} / \mathrm{rev}$; depth of cut: $1 \mathrm{~mm}$. Workpiece material: $40 \mathrm{Cr}$ steel; tool material: high-speed-steel, without coolant. 


\section{Fuzzy neural network}

Suppose the input and output pairs are $\mathrm{X}=\left(x_{1}, x_{2}, \ldots, x_{n}\right)$ and $\mathrm{Y}=\left(y_{1}, y_{2}, \ldots, y_{m}\right)$, respectively. Based on fuzzy inference, $\mathrm{Y}$ is determined by $\mathrm{X}$ and $\mathrm{W}$ as follows:

$$
\mathrm{Y}=\mathrm{X} \cdot \mathrm{W}
$$

where $y_{j}=\max \left(\min \left(x_{i}, w_{i j}\right)\right)(i=1,2, \ldots, n ; j=1,2, \ldots, m), \mathrm{X} \in[0,1], \mathrm{Y} \in[0,1] . w_{i j}$ is the elements of the weight matrix $\mathrm{W}$.

Assuming that the desired output value is $T_{j}$, the actual value is $O_{j}$, the minimizing the square of the difference between them is $E$ :

$$
E=-\frac{1}{2}\left(T_{j}-O_{j}\right)
$$

where: $O_{j}=\max \left(\min \left(x_{i}, w_{i j}\right)\right)$,

It is well known that

$$
\frac{\partial O_{j}}{\partial w_{i j}}=\frac{\partial E}{\partial O_{j}} \cdot \frac{\partial O_{j}}{\partial w_{i j}}
$$

where:

$$
\frac{\partial O_{j}}{\partial w_{i j}}=\frac{\partial \bigvee\left(\bigwedge\left(x_{i}, w_{i j}\right)\right)}{\partial \wedge\left(x_{s}, w_{s j}\right)} \cdot \frac{\partial \wedge\left(x_{s}, w_{s j}\right)}{\partial w_{s j}}
$$

Set:

$$
a_{1}=\frac{\partial \bigvee\left(\wedge\left(x_{i}, w_{i j}\right)\right)}{\partial \wedge\left(x_{s}, w_{s j}\right)}=\frac{\partial \bigvee\left(\bigwedge\left(x_{s}, w_{s j}\right), \bigvee_{i \neq s}\left(\bigwedge\left(x_{i}, w_{i j}\right)\right)\right)}{\partial \wedge\left(x_{s}, w_{s j}\right)} a_{2}=\frac{\partial \wedge\left(x_{s}, w_{s j}\right)}{\partial w_{s j}}
$$

will respond to $a_{1}, a_{2}$ values as follows:

when $\wedge\left(x_{s}, w_{s j}\right) \geq \underset{i \neq s}{\bigvee}\left(\bigwedge\left(x_{i}, w_{i j}\right)\right), a_{1}=1$, otherwise $a_{1}=\wedge\left(x_{s}, w_{s j}\right)$;

when $x_{s} \geq w_{s j}, a_{2}=1$ otherwise $a_{2}=x_{s}$.

Assuming

$$
\frac{\partial O_{j}}{\partial w_{s j}}=\Delta
$$

According to fuzzy min-max inference and smooth derivative theory, fuzzy ruler is as follows:

$$
\begin{aligned}
& \text { if } x_{s}<w_{s j} \text { and } x_{s} \geq \bigvee_{i \neq s}\left(\wedge\left(x_{i}, w_{i j}\right)\right) \text { then } \Delta=x_{s} \\
& \text { if } x_{s}<w_{s j} \text { and } x_{s}<\bigvee_{i \neq s}\left(\bigwedge\left(x_{i}, w_{i j}\right)\right) \text { then } \Delta=x_{s}^{2} \\
& \text { if } x_{s} \geq w_{s j} \text { and } w_{s j} \geq \bigvee_{i \neq s}\left(\wedge\left(x_{i}, w_{i j}\right)\right) \text { then } \Delta=1 \\
& \text { if } x_{s} \geq w_{s j} \text { and } w_{s j}<\bigvee_{i \neq s}\left(\bigwedge\left(x_{i}, w_{i j}\right)\right) \text { then } \Delta=w_{s j}
\end{aligned}
$$


and

$$
\frac{\partial E}{\partial O_{j}}=-\left(T_{j}-O_{j}\right)
$$

Set:

$$
\delta=-\frac{\partial E}{\partial O_{j}}
$$

then $\mu=0.8$

$$
\frac{\partial E}{\partial w_{i j}}=\delta_{j} \Delta
$$

the changes of the weight will be obtained from a $\delta$-rule with expression

$$
\Delta w_{i j}=\mu \delta_{j} \Delta
$$

where $\mu$ is learning rates, $\mu \in[0,1]$.

Under the same condition (training sample, structure, learning rate $\mu=0.8$, convergence error), the FNN training iteration is 8 , and the BPNN is 427 . Hence FNN is a higher effective neural network in comparison with BPNN.

In the paper, the inputs of FNN are above seven monitoring features of AE, which are extracted by wavelet packet transform. The outputs of FNN are the six-membership degree of tool wear condition.

\section{Experimental set-up and multi-parameter fusion}

The schematic diagram of the experimental set-up is shown in Fig. 7(a). Cutting tests were performed on Machining Center Makino-FNC74-A20. In the experiments, a commercial piezoelectric AE transducer made in Shenyang Acoustic Instrument Co. was mounted beside the spindle. AE signals were transmitted by magnetic fluid from the spindle to the transducer as shown in

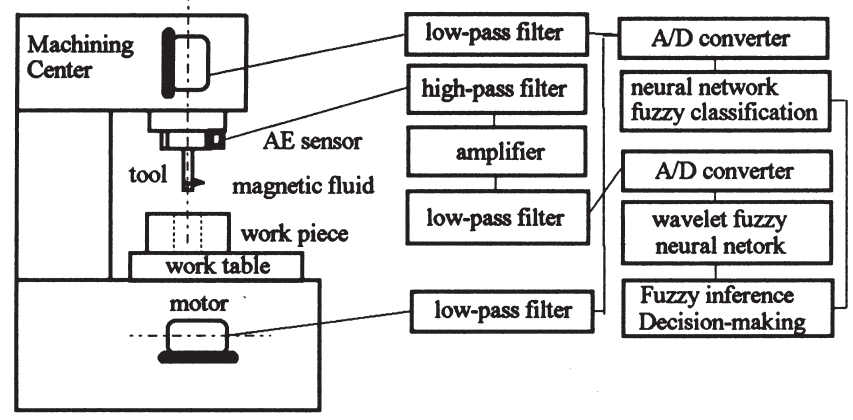

(a)

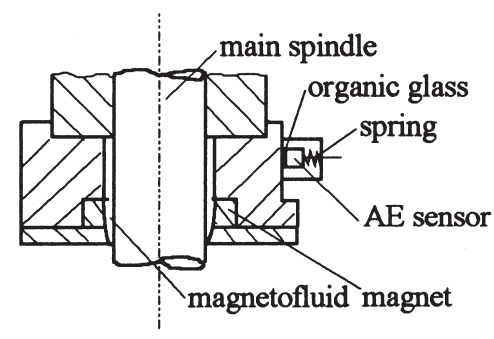

(b)

Fig. 7. Schematic diagram of the experimental set-up. 
Table 3

Experimental conditions for the boring example

Tool

Cutting condition

Bore, high-speed steel; tool geometry: $\gamma=10^{\circ}, \alpha=8^{\circ}$,

$\lambda=-2^{\circ}, \chi=90^{\circ}, \kappa=12^{\circ}$, and $r=0.3 \mathrm{~mm}$
Cutting speed: $20-40(\mathrm{~m} / \mathrm{min})$; feed rate: $0.1,0.2,0.3$

$(\mathrm{mm} / \mathrm{r})$; the depth of cutting: $0.1,0.2,0.5,0.75,1.0,1.25$

(mm); without coolant

Workpiece

45\# quenching-and-tempering steel

Fig. 7(b). During the experiments, the monitored AE signals were amplified and filtered with a band-pass filter, high-passed at $50 \mathrm{kHz}$ and low-passed at $1 \mathrm{MHz}$, and then were sent via an A/D converter to a personal computer. The AE detection system was calibrated and verified by standard $\mathrm{AE}$ signals generated at the tool holder in the spindle with an $\mathrm{AE}$ generator provided by the sensor manufacturer.

A successful tool wear detecting method must be sensitive to tool wear changes and insensitive to the variation of cutting conditions. Hence, cutting tests were conducted at different conditions to evaluate the performance of the proposed method. The tool parameters and cutting conditions are listed in Table 3.

The membership degree of tool wear classification had been calculated by fuzzy classification with spindle current and feed current and using wavelet FNN with AE. The above three parameters would be fused by fuzzy inference to obtain the tool wear value accurately.

The key to the fusion of tool wear states is the selection of appropriate shapes of the fuzzy membership. Here, a trapezoid shape is chosen, and the trapezoid function is defined as follows:

$$
\mu(w)=a w+b \quad k<w<l
$$

Where: $\mu(w)$ is the fuzzy membership value for tool wear states, and $a, b, k$ and $l$ are constants for difference fuzzy sets as shown in Fig. 8.

The relationship between input and output variables of the fuzzy system is defined by a set of linguistic statements that are called fuzzy rules. In the paper, there are three input variables each of which is classified into six fuzzy sets and an output variable is also classified into six fuzzy sets, based on the experimental work, 26 rules are set. These rules are classified into six groups corresponding to six tool wear states.

By using a fuzzy min-max algorithm, i.e. fuzzy intersection (AND) and fuzzy union (OR), the following equation can be generated to calculate the fuzzy membership values for tool wear states:

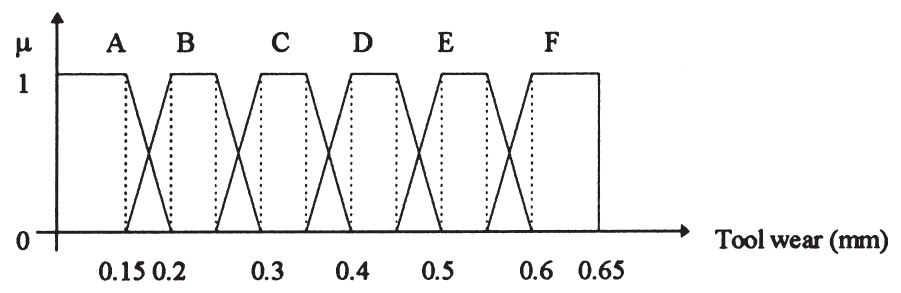

Fig. 8. Fuzzy membership functions of tool wear states. 


$$
\mu_{i}(w)=\bigcup_{j=1}^{K}\left\{\mu_{i}^{s}(w) \cap \mu_{i}^{F}(w)\right\}
$$

where $\mu_{i}(w)(i=\mathrm{A}, \mathrm{B}, \ldots, \mathrm{F})$ is the fuzzy membership for tool wear states under the $\mathrm{A}, \mathrm{B}, \mathrm{C}, \mathrm{D}$, $\mathrm{E}, \mathrm{F}$ classification and $j=1,2, \ldots, K$ represents the number of rules fired for the corresponding tool wear states.

The outputs of the inference process are still fuzzy values and they need to be defuzzified. Basically defuzzification is a mapping from a space of the fuzzy values into that of an non-fuzzy universe. At present, there are several strategies that can be used to perform the defuzzification process. The most commonly used strategy is the centered defuzzy method, which produces the center of the area of the probability distribution of the inference output. So the defuzzified tool wear states can be obtained by using the following centered formula:

$$
\text { wear }=\frac{\int_{w}[\mu(w) w] \mathrm{d} w}{\int_{w} \mu(w) \mathrm{d} w}
$$

where 'wear' represents the numerical value of tool wear and $\mu(w)$ is the fuzzy membership degree fused by fuzzy inference.

\section{Result and discussion}

A total of 77 tool wear cutting tests were collected under various cutting conditions. Fifty samples were randomly picked as learning samples; 27 samples were used as the test samples in the classification phase.

The above method is used to estimate the tool wear value. The membership degree of present tool states under different tool wear classifications is calculated, which is fused by fuzzy inference. The accurate tool wear value is detected using the centered defuzzy method. In order to make clear the reliability of the above method the comparison of actual tool wear values estimated is shown in Fig. 9. The results show that the above method can more accurately estimate tool wear states.

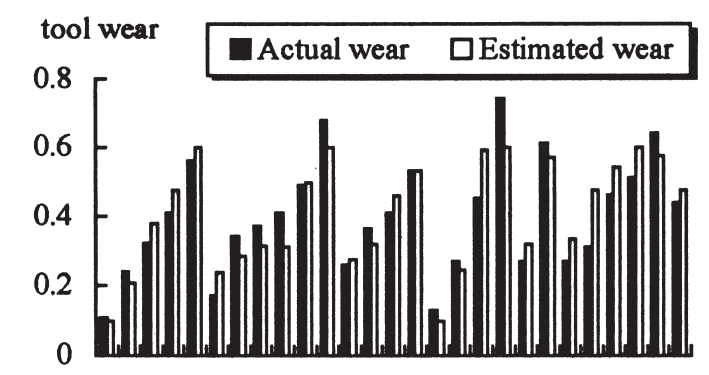

Fig. 9. The comparison of actual wear with estimated wear. 


\section{Conclusion}

In the paper, a new method of tool wear states detection is presented. It can effectively detect tool wear values with detected current signals and AE signal. The following conclusions are drawn:

1. Models of the relationship between the current signals and the cutting parameters under different tool wear states are established over different cutting conditions using experimental design and neural network with regression analysis. The fuzzy classification method has been successfully used to calculate the membership under different tool wear states with detected current signals.

2. The wavelet packet transform is a powerful tool of signal processing in the tool wear detection system. It can capture improvement features of the sensor signal, namely, features are sensitive to the change of tool wear condition, but are insensitive to the variation of process working conditions and various noises. The RMS of the wavelet coefficient of the components selected can be considered as the monitoring features. The pretreated monitoring features have low sensitivity to changes in the process variables. The feature extracted with the wavelet packet transform can be implemented in real time as the wavelet packet transform requires only a small amount of computation.

3. The fuzzy relationship between the tool wear states and monitoring indices may be identified by use of a fuzzy neural network. The training speed of FNN is faster than a back propagation type (BP) neural network.

4. Fuzzy inference is used to fuse the membership degree of tool wear states obtained from spindle, feed current and AE signal. The tool wear value is finally obtained. Experimental results show that the method can be effectively employed in practice.

\section{Acknowledgements}

The support of this work by the China National Defense Foundation (1995-1997) is gratefully acknowledged.

\section{References}

[1] G. Byrne, D. Dornfeld, I. Inasaki, G. Ketteler, W. Konig, R. Teti, Tool condition monitoring (TCM)—-the status of research and industrial application, Annals of the CIRP 44 (2) (1995) 541-567.

[2] D.A. Dornfeld, Neural network sensor fusion for tool condition monitoring, Annals of the CIRP 39 (1990) $101-105$.

[3] K. Iwata, T. Moriwaki, An application of acoustic emission to in-process sensing of tool wear, Annals of the CIRP 26 (1977) 21-26.

[4] S. Liang, D.A. Dornfeld, Tool wear detection using time series analysis of acoustic emission, Transactions ASME, Journal of Engineering and Industry 111 (2) (1989) 199-204.

[5] J.M. Lee, D.K. Choi, C.N. Chu, Real-time tool breakage monitoring for NC turning and drilling, Annals of the CIRP 43 (1994) 81-84. 
[6] I.N. Tansel, C. Mekdeci, O. Rodriguez, B. Uragun, Monitoring drill conditions with wavelet based encoding and neural network, International Journal of Machine Tools Manufacture 33 (4) (1993) 559-575.

[7] YA Wu, R. Du, Feature extraction and assessment using wavelet packets for monitoring of machining process, Mechanical Systems and Signal Processing 10 (1) (1996) 29-53.

[8] XIAOLI Li, YINGXUE Yao, ZHEJUN Yuan, On-line tool condition monitoring using wavelet fuzzy neural network, Journal of Intelligent Manufacturing 8 (1997) 271-276.

[9] I. Daubechies, The wavelet transform, time-frequency localization and signal analysis, IEEE Transactions on Information Theory 36 (5) (1990) 961-1005.

[10] M.S. Lan, D.A. Dornfeld, Experimental studies of flank wear via acoustic emission analysis, in: 10th NAMRC Proceedings, 1982, pp. 305-311. 